\title{
THE WORD PROBLEM FOR FINITELY PRESENTED MONOIDS AND FINITE CANONICAL REWRITING SYSTEMS
}

\author{
Craig Squier \\ Department of Mathematics, State University of New York \\ Binghamton, N.Y. 13001, U.S.A. \\ Friedrich Otto \\ Fachbereich Informatik, Universitaet Kaiserslautern, Postfach 3049 \\ 6750 Kaiserslautern, Fed. Rep. Germany \\ currently visiting at \\ Department of Computer Science, State University of New York \\ Albany, N.Y. 12222, U.S.A.
}

\begin{abstract}
The main purpose of this paper is to describe a negative answer to the following question:

Does every finitely presented monoid with a decidable word problem have a presentation $(\Sigma ; R)$ where $R$ is a finite canonical rewriting system?

To obtain this answer a certain homological finiteness condition for monoids is considered. If $M$ is a monoid that can be presented by a finite canonical rewriting system, then $M$ is an $(F P)_{3}$-monoid. Since there are well-known examples of finitely presented groups that have easily decidable word problem, but that do not meet this condition, this implies that there are finitely presented monoids (and groups) with decidable word problem that cannot be presented by finite canonical rewriting systems.
\end{abstract}

\section{Introduction}

Let $\Sigma$ be an alphabet. Then $\Sigma *$ denotes the free monoid generated by $\Sigma$ with identity 1 , the empty word. A string rewriting system $R$ on $\Sigma$ is a subset of $\Sigma^{*} \times \Sigma^{*}$, the elements of which are called (rewrite) rules. $R$ induces a single-step reduction relation $\Rightarrow_{R}$ on $\Sigma^{*}$, which is defined through $u \Rightarrow_{R} y$ if and only if $u=x l y$ and $v=x r y$ for some words $x, y \in \Sigma^{*}$ and a rule $(l, r) \in R$. The reflexive transitive closure $\Rightarrow_{R}^{*}$ of $\Rightarrow_{R}$ is the reduction relation generated by $R$, and the reflexive symmetric and transitive closure $\leftrightarrow_{R}^{*}$ of it is the Thue congruence generated by $R$. The factor monoid $\Sigma^{*} / \leftrightarrow_{R}^{*}$ of the free monoid $\Sigma^{*}$ modulo the congruence $\leftrightarrow{ }_{R}$ is denoted by $M_{R}$, and the ordered pair $(\Sigma, R)$ is called a monoid presentation of this monoid.

The word problem for $M_{R}$ is the following well-known decision problem : 
INSTANCE : Two words $u, v \in \Sigma^{*}$.

QUESTION : Do $u$ and $v$ represent the same element of $M_{R}$, i.e., does $u \leftrightarrow{ }_{R}^{*} v$ hold?

Although the word problem is defined using a particular presentation, its decidability and intrinsic complexity are independent of the actually chosen presentation as long as this presentation is finitely generated, i.e., $\Sigma$ is finite [1].

The rewriting system $R$ is called canonical or complete, if the process $\Rightarrow_{R}$ of reduction modulo $R$ is Noetherian and confluent. In this situation $R$ defines a unique normal form for each of the congruence classes of $\leftrightarrow R_{R}^{*}$. Thus, if the process of reduction is an effective process, which is in particular the case if $R$ is finite, then the word problem for $M_{R}$ can be solved by the following normal form algorithm :

INPUT : $u, v \in \Sigma^{*}$

begin reduce $u$ to its normal form $u_{0}$;

reduce $v$ to its normal form $v_{0}$;

if $u_{0}=v_{0}$ then ACCEPT else REJECT

end.

This algorithm is a pseudo-natural algorithm in the sense of [17], since in the affirmative it not only gives the correct answer, but it also gives a proof for this answer in the form of reduction sequences from $u$ and $v$ to their common normal form. The derivational complexity $f_{R}$ of $R$, which is defined as $f_{R}(u, v):=\min \left\{i \mid \exists x_{1}, x_{2}, \ldots, x_{i-1} \in \Sigma^{*}\right.$ : $\left.u \leftrightarrow_{R} x_{1} \leftrightarrow_{R} x_{2} \leftrightarrow_{R} \ldots \leftrightarrow_{R} x_{i-1} \leftrightarrow_{R} v\right\}$, induces a lower bound for the complexity of this normal form algorithm.

Here we are interested in the relationship between the decidability of the word problem for a finitely presented monoid, its complexity, and presentations by finite canonical rewriting systems. Let $M_{R}$ be a monoid with a decidable word problem given through a finite presentation $(\Sigma ; R)$. We are going to present answers for the following questions:

(1) If $R$ is canonical, is there an a-priori upper bound for the intrinsic complexity of the word problem?

(2) If $R$ is canonical, is there an a-priori upper bound for the complexity of the corresponding normal form algorithm, and therewith for the derivational complexity $f_{R}$ of $R$ ?

(3) If $R$ is not canonical, does there exist a finite canonical system $R_{1}$ on $\Sigma$ such that $R$ and $R_{1}$ generate the same Thue congruence on $\Sigma^{*}$, i.e., $R$ and $R_{1}$ are equivalent ?

(4) If $R$ is not canonical, does there at least exist a finite canonical system $S$ on alphabet $\Gamma$ such that the monoids $M_{R}$ and $M_{S}$ are isomorphic, i.e., $(\Gamma ; S)$ is another presentation of $M_{R}$ ?

A positive answer to (4) would imply that there always exists a finite presentation of $M$ such that using this presentation the word problem for $M$ can be solved by a normal form algorithm of the form described above. 
After summarizing the results for questions (1) to (3) we shall describe the negative answer for question (4) recently obtained by Squier [20]. Because of this negative result we are then led to ask about the possibility of presenting finitely generated monoids that have a decidable word problem through infinite canonical rewriting systems for which the operation of reduction is easily computable.

Complexity results will be stated using the Grzegorczyk hierarchy $E_{n}(n \geq 0)[11,22]$, but other sufficiently rich complexity classes could be used as well.

\section{Previous results}

Because of the close correspondence between Turing machines and string rewriting systems it is well-known that there exist finitely presented monoids with word problems of arbitrary degree of complexity. In his dissertation [2] Bauer proved that this situation does not change when monoids are considered that are presented by finite canonical rewriting systems.

Theorem $1[2,4]$. Let $m$ be an integer with $m>1$. Then there exists a finite canonical rewriting system $R$ on alphabet $\Sigma$ such that the word problem for $M_{R}$ is of intrinsic complexity $E_{m}-E_{m-1}$.

For a finite canonical rewriting system the derivational complexity induces an upper bound for the intrinsic complexity of the word problem, and as mentioned before it induces a lower bound for the complexity of the normal form algorithm. However, in general there is an arbitrarily large gap between the intrinsic complexity of the word problem and the derivational complexity.

Theorem $2[4]$. Let $m, n$ be integers with $3 \leq n<m$. Then there is a finite canonical system $R$ on alphabet $\Sigma$ satisiying the following conditions :

(a) The word problem for $M_{R}$ is of intrinsic complexity $E_{n}-E_{n-1}$.

(b) The derivational complexity $f_{R}$ of $R$ belongs to $E_{m}-E_{m-1}$.

Thus, even when we know that the word problem for a given finite canonical system is of a low degree of complexity, we do not have an a-priori upper bound for the complexity of the corresponding normal form algorithm. Like the intrinsic complexity of the word problem also the derivational complexity is an invariant of finite presentations [17]. Hence, changing the monoid presentation under consideration does not help in this situation. So it appears that even when dealing with finite canonical rewriting systems it might be necessary to develop other types of algorithms for solving the word problem than just the normal form algorithm.

So far we have seen that finite canonical rewriting systems are powerful enough to present monoids with word problems of many different degrees of complexity. Are these systems powerful enough to present all monoids with a decidable word problem? Moreover, does every finite 
presentation $(\Sigma ; R)$ of a monoid $M$ with a decidable word problem allow a finite canonical rewriting system on the given set of generators $\Sigma$, i.e., is there a finite canonical rewriting system $R_{1}$ on $\Sigma$ such that $R$ and $R_{1}$ generate the same Thue congruence?

The following example concerning the last question was given by Kapur and Narendran [14].

Example 1. Let $\Sigma=\{a, b\}$, and let $R=\{(a b a, b a b)\}$. Then the word problem for the monoid $M_{R}$ presented by $(\Sigma, R)$ is decidable, but there does not exist a finite canonical rewriting system $R_{1}$ on $\Sigma$ such that $R$ and $R_{1}$ are equivalent. On the other hand, by introducing a third letter $c$ we obtain the presentation $\left(\{a, b, c\} ; R_{1}\right)$ with $R_{1}=\{(a b, c),(a c, c a)\}$ of $M_{R}$. As can be checked easily $R_{1}$ is canonical.

Hence, we have the following negative answer for this question.

Theorem 3 [14]. There exists a finite rewriting system $R$ on alphabet $\Sigma$ such that the word problem for $M_{R}$ is easily decidable, but there is no finite canonical system $S$ on $\Sigma$ that is equivalent to $R$.

Jantzen gives another example of this phenomenon [12].

Example 2. Let $\Sigma=\{a, b\}$, and let $R=\{(a b b a, 1)\}$. The monoid $M_{R}$ is actually a group, the word problem for which is decidable in linear time [13], but there is no finite canonical rewriting system $R_{1}$ on $\Sigma$ that is equivalent to $R$. However, $R_{1}=$ $\{(c d, 1),(d c, 1),(x y, 1),(y x, 1),(c x, y c),(c y, x c),(d x, y d),(d y, x d)\}$ is a canonical rewriting system on $\Gamma=\{c, d, x, y\}$ such that $\left(\Gamma ; R_{1}\right)$ is another presentation of $M_{R}$.

Finally, by considering monoid presentations of abelian groups Diekert derives a whole class of finite rewriting systems which are not equivalent to finite canonical systems, but for which the word problem can be solved in linear time [10]. For all the systems mentioned so far there exist finite canonical rewriting systems on different alphabets defining the same monoids, i.e., by changing the presentation we obtain a finite canonical rewriting system for each of these monoids.

This observation might be seen as supporting the conjecture that every finitely presented monoid with a decidable word problem does at least have some presentation by a finite canonical system. Also the following result which was obtained by Bauer while he was studying 'nlevel' rewriting systems can be interpreted in this way.

Theorem $4[2,3]$. Let $M$ be a finitely presented monoid with a decidable word problem. Then there exists a finite canonical two-level rewriting system $R$ on some finite alphabet $\Sigma$ such that $(\Sigma ; R)$ is a presentation of $M$. 
Here a wo-level rewriting system $R$ over $\Sigma$ is a pair $\left\langle R_{1}, R_{2}\right\rangle$, where $R_{1}$ and $R_{2}$ are rewriting systems over $\Sigma$ with the following rule for applying rewrite rules:

A sequence of rewrite rule applications of $R$ starting with a word $w \in \Sigma^{*}$ is called admissible if it is of the form $w \Rightarrow_{R_{1}}^{*} w_{1} \Rightarrow_{R_{2}}^{*} w_{2}$, where $w_{1}$ is irreducible modulo $R_{1}$ and $w_{2}$ is irreducible modulo $R_{2} . R$ is canonical if, for each $w \in \Sigma^{*}$, each admissible sequence starting with $w$ ends after a finite number of steps with the same normal form $w_{0}$ of $w$.

Question (A) asks whether or not Theorem 4 ean be improved to always yield finite (onelevel) canonical rewriting systems. In fact much work has been directed towards finding finite canonical systems for various classes of groups with decidable word problem (cf., e.g., [16]).

\section{A homological finiteness condition}

Question (4) bas attracted quite some attention in recent years $[4,7,12]$. The following result now answers this question in the negative.

Theorem 5 [20]. There exist fnitely presented monoids $M$ (in fact, even groups) having the following properties:

(a) The word problem for $M$ is decidable.

(b) $M$ does not allow a presentation of the form $(\Sigma ; R)$, where $R$ is a finite canonical rewriting system on $\Sigma$.

Thus, not all finitely presented monoids and groups with a decidable word problem can be presented by finite canonical rewriting systems. At this point it remains open exactly which monoids and groups with a decidable word problem can be presented in this way.

The proof of Theorem 5 consists in two steps. First an intrinsic property for monoids that are presented by finite canonical systems is established, and then examples of finitely presented monoids with decidable word problem that do not satisfy this property are exhibited. This intrinsic property is a homological finiteness condition.

Theorem 6. Let $R$ be a finite canonical rewriting system on $\Sigma$. Then the monoid $M_{R}$ presented by $(\Sigma ; R)$ is an $(F P)_{3}$-monoid.

We remark that the property of being an $(F P)_{3}$-monoid is a property of monoids rather than of presentations. For sake of completeness we now give the definition of the property $(F P)_{k}$.

Let $M$ be a monoid. Then $Z M$ denotes the integral monoid-ring of $M$. Also we view the integers $Z$ as a left $Z M$-module, where each element of $M$ acts on $Z$ as multiplication by 1 . 
Now $M$ is said to be an $(F P)_{k}-$ monoid $(k \geq 1)$, if there is an exact sequence

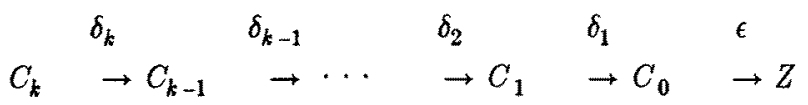

of finitely generated free left $Z M$-modules $C_{k}, C_{k-1}, \ldots, C_{0}$ and $Z M$-module homomorphisms $\delta_{k}, \delta_{k-1}, \ldots, \delta_{1}, \epsilon$. The notion of $(F P)_{k}$-group is well-known (cf., e.g., $[5,8]$ ).

Throughout the following we can assume that $C_{0}$ is a free left $Z M$-module generated by a single element.

If a monoid $M$ is finitely generated, then $M$ is an $(F P)_{1}$ - monoid, since the generators of the free left $Z M$-module $C_{1}$ can be chosen to correspond to a set of generators of $M$. For groups the converse implication also holds: If a group $G$ is $(F P)_{1}$, then $G$ is finitely generated. However, for monoids in general this does not hold.

Example 3. Let $\Sigma=\{y\} \cup\left\{x_{i} \mid i \in N\right\}$, and $R=\left\{\left(x_{i+1} y, y x_{i}\right) \mid i \in N\right\}$. Then the monoid $M_{R}$ presented by $(\Sigma ; R)$ is not finitely generated, but it is an $(F P)_{1}$-monoid. In fact, $M_{R}$ is an $(F P)_{k}$-monoid for each $k \geq 1$.

If a monoid $M$ is finitely presented, then $M$ is an $(F P)_{2}$ - monoid, since the generators of the free left $Z M$-module $C_{2}$ can be chosen to correspond to a set of defining relations of $M$. It is an open question whether each $(F P)_{2}$-group is finitely presented; for monoids in general it does not hold as indicated by Example 3. However, the monoid $M_{R}$ presented in Example 3 is not even finitely generated. We do not know at this time whether there exists a finitely generated $(F P)_{2}$-monoid that is not finitely presented.

Now the idea behind Theorem 6 is the following: Let $R$ be a canonical system on alphabet $\Sigma$ such that $(\Sigma ; R)$ is a presentation of $M$. Then a set of generators for the free left $Z M$-module $C_{3}$ can be chosen to correspond to the set of critical pairs of $R$. If $R$ is finite, then the set of critical pairs of $R$ is also finite, and so $C_{3}$ is finitely generated. Combining the above we obtain Theorem 6.

To complete the proof of Theorem 5 it suffices to exhibit a finitely presented monoid $M$ with a decidable word problem such that $M$ is not $(F P)_{3}$. The first example of a finitely presented group which is not an $(F P)_{3}$-group is due to Stallings [21]. A description of this group which makes it clear that this group has a decidable word problem can be found in Bieri's monograph [5]. Further examples of finitely presented groups that are not $(F P)_{3}$-groups are given in $[6,9]$.

For completeness we give an example of a finitely presented monoid with a decidable word problem such that this monoid is not $(F P)_{3}$.

Example 4. For each non-negative integer $k$, let $S_{k}$ denote the monoid defined by the presentation $\left(\Sigma_{k} ; R_{k}\right)$, where $\Sigma_{k}=\left\{a, b, t, x_{1}, x_{2}, \ldots, x_{k}, y_{1}, y_{2}, \ldots, y_{k}\right\}$, and $R_{k}=\left\{\left(a t^{n} b, 1\right) \mid n \geq 0\right\} \cup$ $\left\{\left(x_{i} a, a t x_{i}\right),\left(x_{i} t, t x_{i}\right),\left(x_{i} b, b x_{i}\right),\left(x_{i} y_{i}, 1\right) \mid i=1,2, \ldots, k\right\}$. 
Although for each $k$ the set of defining relations $R_{k}$ is infinite, one can verify easily that for all $k \geq 1$, the monoid $S_{k}$ has a finite presentation. On the other band, for each $k$, the system $R_{k}$ is canonical, and due to the simplieity of the rules of $R_{k}$ the reduction process $\Rightarrow_{R_{k}}$ is effective, showing that the word problem for the monoid $S_{k}$ is decidable. In fact, it is decidable in polynomial time $\left(E_{2}\right)$. However, for $k \geq 2$, the monoid $S_{k}$ is not an $(F P)_{3}$-monoid. Thus, these monoids cannot be presented by finite canonical rewriting systems (cf. [20] for details).

In the above example $S_{1}$ is an $(F P)_{3}$-monoid. We do not know whether or not $S_{1}$ can be presented by a finite canonical system. Actually, it is a general question whether the property of being an $(F P)_{3}$-monoid is not only necessary, but also sufficient for a finitely presented monoid with a decidable word problem to have a presentation by some finite canonical rewriting system. We conjecture that this condition is not sufficient.

\section{Concluding remarks}

Each monoid $M$ with a decidable word problem can be presented by an infinite canonical rewriting system. Let $(\Sigma ; R)$ be a presentation of $M$, and let $<_{M}$ denote a well-ordering on $\Sigma^{*}$ that is compatible with the operation of concatenation. For $w \in \Sigma^{*}$, let $w_{0}$ denote the minimal element in the congruence class of $w$ with respect to this ordering. Then $S=\left\{\left(w, w_{0}\right) \mid\right.$ $\left.w \in \Sigma^{*}, w \neq w_{0}\right\}$ is a canonical rewriting system on $\Sigma$ that is equivalent to $R$. However, in general this system is not very helpful for actually solving the word problem, since the problem of deciding whether a given pair of words $(l, r)$ is a rule of $S$ is already as complex as the word problem itself. Thus, infinite canonical rewriting systems will only be useful for actually solving the word problem, if they are sufficiently simple. Therefore we are led to the following two questions:

(5) For every finitely presented monoid $M$ with a decidable word problem, does there exist an infinite canonical rewriting system $R$ on some alphabet $\Sigma$ such that $(\Sigma ; R)$ presents $M, R$ has a regular (or context-free, or ...) set of left-hand sides, and the process of reduction $\Rightarrow_{R}$ is easily computable?

(6) In the setting above, can $R$ always be chosen in such a way that the derivational complexity $f_{R}$ and the intrinsic complexity of the word problem are closely related?

Each of the systems $R_{k}$ in Example 4 has a regular set of left-hand sides, and the induced reduction relation $\Rightarrow_{R_{k}}$ is easily computable. Different kinds of regular canonical rewriting systems have been investigated by Kemmerich [15] and O'Dunlaing [19], and context-free canonical rewriting systems have been investigated by Book, Jantzen, and Wrathall [8].

The corresponding questions for non-canonical rewriting systems have been solved in the affirmative [18]. The derivational complexity for a non-canonical rewriting system also induces an upper bound for the intrinsic complexity of its word problem. The complexity gap of 
Theorem 2 also occurs in this more general situation [17]. Even if we consider rewriting systems $R$ such that the language $L_{R}=\{u \# v \mid(u, v) \in R\}$ is context-free, the situation does not change [18]. However, once we deal with rewriting systems $R$ for which the language $L_{R}$ is context-sensitive, the situation is different.

Theorem 7 [18]. Let $M$ be a finitely generated monoid. If the word problem for $M$ is decidable of complexity $E_{n}$ for some positive integer $n$, then there exists a rewriting system $R$ on alphabet $\Sigma$ satisfying the following properties :

(a) $(\Sigma ; R)$ is a presentation of $M$.

(b) The language $L_{R}$ is context-sensitive.

(c) The derivational complexity $f_{R}$ belongs to $E_{n}$.

Thus, when allowing rewriting systems $R$ such that the languages $L_{R}$ are context-sensitive, then the derivational complexity can be made to meet the intrinsic complexity of the word problem. So questions (5) and (6) asks whether these results can be extended to canonical rewriting systems.

Notice that question (4) can be asked for all kinds of combinatorial systems that present algebraic structures. For example it is known that every finitely generated abelian group can be presented by a finite canonical string rewriting system [10]. However, for most combinatorial systems and algebraic structures this question is still open.

\section{References}

1. J. Avenhaus, K. Madlener; Subrekursive Komplexitaet bei Gruppen; I. Gruppen mit vorgeschriebener Komplexitaet; Acta Informatica 9 (1977), 87-104.

2. G. Bauer; Zur Darstellung von Monoiden durch konfluente Regelsysteme; dissertation, Fachbereich Informatik, Universitaet Kaiserslautern, 1981.

3. G. Bauer; N-level rewriting systems; Theoret. Comput. Science 40 (1985), 85-99.

4. G. Bauer, F. Otto; Finite complete rewriting systems and the complexity of the word problem; Acta Informatica 21 (1984), 521-540.

5. R. Bieri; Homological dimension of discrete groups; Queen Mary College Mathematics Notes, London, 1976.

6. R. Bieri; A connection between the integral homology and the centre of a rational linear group; Math. Z. 170 (1980), 263-266.

7. R.V. Book; Thue systems as rewriting systems; in J.P. Jouannaud (ed.), Rewriting Techniques and Applications, Lecture Notes Comp. Science 202 (1985), 63-94.

8. R.V. Book, M. Jantzen, C. Wrathall; Monadic Thue systems; Theoret. Comput. Science 19 (1982), 231-251.

9. K.S. Brown; Finiteness properties of groups, preprint.

10. V. Diekert; Complete semi-Thue systems for abelian groups; Theoret. Comput. Science 44 (1986), 199-208.

11. A. Grzegorczyk; Some classes of recursive functions; Rozprawy Math. 4 (1953), 1-45.

12. M. Jantzen; A note on a special one-rule semi-Thue system; Inf. Proc. Letters 21 (1985), 135-140.

13. M. Jantzen; Thue congruences and complete string rewriting systems; Habilitationsschrift; Fachbereich Informatik, Universitaet Hamburg, 1986.

14. D. Kapur, P. Narendran; A finite Thue system with decidable word problem and without equivalent finite canonical system; Theoret. Comput. Science 35 (1985), 337-344.

15. S. Kemmerich; Unendliche Reduktionssysteme; dissertation, Fachbereich Mathematik, TH 
Aachen, 1983.

16. $\mathrm{Ph}$. LeChenadec; Canonical Forms in Finitely Presented Algebras; Pitman, London, John Wiley \& Sons,Inc., New York - Toronto, 1986.

17. K. Madlener,F. Otto; Pseudo-natural algorithms for the word problem for finitely presented monoids and groups; J. Symbolic Comput. 1 (1985), 383-418.

18. K. Madlener, F. Otto; Pseudo-natural algorithms for finitely generated presentations of monoids and groups; J. Symbolic Comput., to appear.

19. C. O'Dunlaing; Infinite regular Thue systems; Theoret. Comput. Science 25 (1983), 171-192.

20. C.C. Squier; Word problems and a homological finiteness condition for monoids; J. Pure Appl. Algebra, to appear.

21. J.R. Stallings; A finitely presented group whose 3-dimensional integral homology is not finitely generated; American J. of Mathematics 85 (1963), 541-543.

22. K. Weihrauch; Teilklassen primitiv-rekursiver Wortfunktionen; Report No. 91, GMD Bonn, 1974. 